\title{
Using paradata to explore item level response times in surveys
}

\author{
Mick P. Couper \\ University of Michigan, Ann Arbor, and University of Maryland, College Park, USA \\ and Frauke Kreuter \\ University of Maryland, College Park, USA, Institut für Arbeitsmarkt- und Berufsforschung, \\ Nürnberg, and Ludwig-Maximilians-Universität München, Germany
}

[Received March 2011. Final revision January 2012]

\begin{abstract}
Summary. We analyse item level keystroke data from cycle 6 of the National Survey of Family Growth, which is a survey on fertility and related topics that is conducted in the USA. The National Survey of Family Growth is conducted among both males and females by using computer-assisted personal visit interviews and an audio computer-assisted self-interviewing component for the most sensitive topics. Our analyses focus on the time taken to answer a question as a function of item level characteristics, respondent characteristics and interviewer characteristics. Using multilevel models, we explore how these factors influence response times. Our exploratory study suggests that factors at all three levels (item, respondent and interviewer) influence response times. These results demonstrate that question features that explain variation in response times can be automatically derived from standard computer-assisted personal interviewing paradata. The effects of respondent characteristics that we observe are in line with prior findings from more controlled studies conducted in supervised telephone facilities. Some demographic characteristics of interviewers contributed to the variation in response times, though they failed to explain large portions of the between-interviewer variance.
\end{abstract}

Keywords: Item level times; Measurement error; Paradata

\section{Introduction}

Paradata are widely used to explore non-response in surveys and to manage data collection (e.g. Kreuter et al. (2010), Kreuter and Kohler (2009) and LaFlamme (2009)). A promising area is the use of paradata-driven responsive design (for example, see Groves and Heeringa (2006)). However, the first uses of paradata focused on keystroke level data to explore measurement error in surveys, particularly in terms of the design of the computer-assisted interviewing (CAI) instruments and interviewer use of computers (e.g. Caspar and Couper (1997) and Couper et al. 1997a,b). Since then, relatively little attention has been paid to keystroke or item level paradata, with the exception of Web surveys where paradata have been used extensively to examine respondents' interaction with the survey instrument (e.g. Heerwegh (2003), Stern (2008) and Yan and Tourangeau (2008)).

The absence of research on the large-scale use of measurement-error-related paradata in interview surveys is unfortunate, given that the audit trail or keystroke data that are generated from CAI systems provide a rich source of information on the process at little additional cost, as was

Address for correspondence: Mick P. Couper, Institute for Social Research, University of Michigan, PO Box 1248, Ann Arbor, MI 48106, USA.

E-mail: mcouper@umich.edu 
argued in the early days of paradata analyses (Couper, 1998). In this paper we explore item level keystroke data, focusing particularly on response time as a variable of interest.

Why is there interest in response time? Measures of time are readily available from most CAI systems including both computer-assisted telephone interviewing (CATI) and computerassisted personal interviewing (CAPI) software. Such measures of time have commonly been used as indicators of possible measurement errors in surveys. Response times were found to be inversely correlated with the tendency to answer positively, irrespectively of the particular item content (Knowles and Condon, 1999; Bassili, 2003) - a phenomenon which is known as acquiescence bias. Shorter response times were found to be associated with context effects reflecting assimilation (Tourangeau, 1992) and the lack of motivation to answer accurately as the survey continues (Bassili, 1996; Yan and Tourangeau, 2008). Heerwegh (2003) found that longer response times were associated with lower scores on knowledge items, and Draisma and Dijkstra (2004) found that longer response times were an indictor of uncertainty and response error and were associated with more non-substantive responses.

Given these findings, a statistical process control perspective on the use of paradata in surveys (see Kreuter et al. (2010)) would suggest using unusually long or unusually short response times as proxy indicators for measurement error, controlling for other factors that influence response times. Past research on response times has identified such factors on the item and respondent level (for a detailed review see Mayerl and Urban (2008)). Studies have found that both the length of the question (Wagner-Menghin, 2002; Yan and Tourangeau, 2008) and its complexity (Bassili and Scott, 1996; Bassili and Krosnick, 2000; Yan and Tourangeau, 2008) increase response times. Practice with survey items, such as the repeated use of the same answer scale or batteries of similar items, have been found to reduce response times (Smith, 1968; Smith and Lerner, 1986; Bassili, 1996; Huckfeldt et al., 1998).

With regard to respondent characteristics, older respondents and those with lower levels of education have been repeatedly found to take longer to answer questions (e.g. Faust et al. (1999), Fisher and Glaser (1996) and Yan and Tourangeau (2008)). Respondents with readily available attitudes exhibited faster response times (Fazio, 1986, 1990; Fazio and Williams, 1986; Ajzen et al., 1995) than those that required processing of a survey question before an answer can be given (Smith et al., 1996). Stable effects of gender on response times have not been found (Mayerl and Urban, 2008). All studies report variation in respondents' baseline speed (Fazio, 1990; Mayerl et al., 2005), which is a variance component that is important to consider when analysing response times.

In large-scale interview surveys, one additional factor, beyond item and respondent characteristics, needs to be taken into account: the interviewer who is responsible for administering the survey. Little is known about interviewer effects on paradata time measures, in part, because a large portion of the response time research has been conducted in selfadministered modes with no interviewer present or measures of response time were focused on the respondent only. There is, however, good reason to assume that interviewers do have an effect on response times. Interviewers vary naturally in their rate of speech and in the way that they react to the respondents' speech patterns (Schaeffer and Maynard, 2006; Conrad et al., 2010). In doing so, interviewers might (consciously or not) influence respondents' rate of speech and their response times. Mayerl and Urban (2008) showed, for example, that respondents increased their response times in response to explicit instructions on speed and accuracy that they were given. Interviewers may not directly give such instructions but signal preferences through their behaviour (Cannell et al., 1981). Interviewer variation in overall survey administration time has also been studied by survey methodologists (see for example Olson and Peytchev (2007)). 
To date, most response time studies have been based on CATI surveys, self-administered Web surveys or self-administered surveys in laboratory settings. In some of these studies response times have been actively captured by the interviewers. With the increase in the use of laptop computers for large-scale face-to-face surveys, item administration times are now available on a much broader scale and are captured automatically in the systems. Such item level times can be used as a proxy for the time that respondents take to answer the question.

Understanding, and potentially controlling for, the interviewer contribution to question administration times is important because the time measures that are automatically captured through paradata do not distinguish between the time taken for the interviewer to read the question, the time taken by the respondent to formulate and provide an answer and the time taken by the interviewer to enter the response into the system. Partialing out the contribution of the interviewer in the overall time to administer an item will thus help to isolate effects that are associated with the respondent or the item.

Taking these three factors - item, respondent and interviewer - into account, this paper investigates the possibilities of using automatically captured item times (as part of the paradata from CAI instruments) as indicators of potential problems with survey items. We consider this an exploratory analysis to investigate sources of variation at the item, respondent and interviewer level. In contrast with prior response time studies, which were mostly based on CATI interviews and with few exceptions (Mayerl and Urban, 2008) focused on a small number of key items, our analyses examine all the items that were fielded in a CAPI survey.

Using CAI response time paradata from a large-scale CAPI survey, and taking advantage of the full hierarchical structure of the data set, we address the following questions.

(a) Are specific syntactical features of the instrument associated with longer or shorter response times?

(b) Do item-specific results found in previous studies hold when controlling for the set of respondents answering such items? And, in controlling for items and interviewers, can we replicate the findings about respondent characteristics and response times found in the CATI literature?

(c) Is there a unique interviewer contribution to question administration time? And, if so, to what extent can this contribution be explained through interviewer characteristics?

Our goal is a set of exploratory analyses to identify the utility of item level times captured from paradata to gain insight into the process of administering survey questions, and to identify — and to attempt to account for-variation in times across items, respondents and interviewers. Using a process-control-oriented framework, the results of this research can help to identify potentially problematic questions or types of questions, to obtain more precise estimates of questionnaire length and to identify interviewers who may be having difficulty with the instrument (i.e. slow), administering the items too fast, or even not administering them at all (i.e. implausibly fast).

\section{Sources of data and variables}

The data that we analyse are from cycle 6 of the National Survey of Family Growth (NSFG) that was conducted in 2002-2003 (see Lepkowski et al. (2006)). The survey is based on a national probability sample of women and men 15-44 years of age. Different instruments are used for female and male respondents. Fieldwork was carried out by the University of Michigan's Survey Research Center. In-person interviews were conducted by female interviewers using CAPI. At the end of the interview, audio computer-assisted self-interviewing (ACASI) was used for the most sensitive questions. The interviewer provided the respondent with a headset and turned the 
laptop around so that the respondent could hear and answer the questions directly without the interviewer observing the respondent's answers.

The NSFG cycle 6 was designed as a multiphase survey, using responsive design to reduce non-response bias (Groves and Heeringa, 2006). Across all three phases a response rate of 79\% for females and $78 \%$ for males was achieved. A total of 12571 respondents - 7643 females and 4928 males - were interviewed (Lepkowski et al., 2006).

The questionnaire contained up to 11 different topical sections, including items about pregnancies, cohabitations, contraception, attitudinal questions towards parenthood, health insurance and related topics. The average total interview time was $80 \mathrm{~min}$ for females and $60 \mathrm{~min}$ for males. The average time for the ACASI portion was $20 \mathrm{~min}$. Full documentation of the survey and questionnaires can be found at http://www. cdc.gov/nchs/nsfg/nsfg_cycle6. htm.

Earlier analyses of NSFG response times focused on the ACASI component (see Couper et al. (2009)) which contains roughly a 10th of all items in the questionnaire. In the present analyses we focus on the full survey instrument, including an indicator for the two response modes, CAPI and ACASI. Given that the questionnaires for men and women are quite different, we analyse the data separately by gender.

Both the CAPI and the ACASI portions of the survey were administered using Blaise, which is a CAI system and survey processing tool developed by Statistics Netherlands. Blaise, like other CAI packages, produces trace or audit trail files that capture the time that an interviewer entered and exited each input field or item (Hansen and Marvin, 2001). In addition to the Blaise audit trails, the Blaise data model contains information on the items (fields) in the instrument and their interrelationships (rules) in a text file that can be electronically parsed (see Statistics Netherlands (2002)). From this file we automatically generate item level characteristics that may affect response times.

\subsection{Description of variables and sources of data}

We merged data from four different sources:

(a) the paradata, extracted from the Blaise files,

(b) characteristics of the items, extracted from the Blaise data model,

(c) respondent characteristics from the interview data sets and

(d) interviewer characteristics from a questionnaire administered to all interviewers working on the project.

The distributions of variables that were included in the final multilevel models from each of these sources are listed in Table 1.

The main goal of our exploratory study was to compare the explanatory power of automatically derived question characteristics from the Blaise audit trails and Blaise instrument. Thus we created a set of field level variables, extracted from the Blaise instrument by using an automated SAS script. Each of these characteristics is hypothesized to influence the response time. The field type variable identifies four different types of response that are permissible in Blaise. Fixed choice questions are those where one response is selected from a list (for example yes-no questions), as opposed to questions where multiple responses are possible (e.g. 'Does (NAME) have a physical disability, an emotional disturbance, or mental retardation?'), those that allow for a numeric answer (e.g. 'How old were you when you had your first menstrual period?') and questions with open answers. Although these simplified categorizations do not capture question types as well as manual coding would, they do reflect different cognitive processes that are 
Table 1. Summary of field, respondent and interviewer variables used in the final multilevel models

\begin{tabular}{lcc}
\hline Variable & Description & Females \\
$(\%$ offields $)$ & $\begin{array}{c}\text { Males } \\
(\% \text { of fields })\end{array}$
\end{tabular}

Field characteristics

Word count

Field type

Field sequence number

\section{ACASI}

Flag: interviewer instruction

Flag: fill

Flag: show card

Flag: help

Respondent characteristics

Race

Marital status

Education

Language of interview

Interviewer characteristics

Education

Spanish speaker

Race

Prior CAI experience
Number of words in field (including interviewer instructions, but excluding fills)

1 , fixed choice (single response)

2 , integer

3 , multiple response

4 , open

Counter for where the item was asked in each interview, (1, 1st question; 2,2 nd, etc.)

1 , ACASI (self administered)

0 , CAPI (interviewer administered)

1 , interviewer instruction

0 , no interviewer instruction

1 , field contains fill text

0 , field does not contain fill text

1 , show card for field

0 , no show card

1 , question help available

0 , no question help available

1 , black

2 , other

3, white

1 , married

2, cohabiting

3 , separated, or widowed or divorced

4 , never married

1 , less than high school

2 , high school

3 , some college

4 , college graduate

1, English

0 , Spanish

0 , unknown

1 , high school or less

2 , college

3 , graduate school

1, speaks Spanish

0 , does not speak Spanish

0 , unknown

1 , black

2, other

3 , white

1 , yes

0 , no
Mean: 36.3

Mean: 28.9

58.9

26.9

7.8

6.4

Mean: 302

69.3

17.3

3.9

9.5

Mean: 172

24.4

38.4

75.6

61.6

69.3

57.6

30.7

49.3

50.7

12.9

87.1

24.5

75.5

42.4

38.3

61.7

14.1

85.9

25.8

74.2

$\%$ of respondents

\begin{tabular}{rr}
\hline 21.8 & 20.4 \\
9.1 & 12.3 \\
21.8 & 67.3 \\
39.5 & 24.8 \\
9.8 & 8.2 \\
11.9 & 9.2 \\
38.8 & 57.8 \\
22.6 & 28.0 \\
28.6 & 30.7 \\
28.6 & 25.0 \\
20.0 & 16.2 \\
93.0 & 92.4 \\
7.0 & 7.6
\end{tabular}

$\%$ of interviewers

$\begin{array}{rr}4.1 & 4.1 \\ 40.1 & 39.6 \\ 40.1 & 40.1 \\ 15.7 & 16.2 \\ 24.4 & 25.0 \\ 75.6 & 75.0 \\ 3.1 & 3.1 \\ 25.9 & 25.5 \\ 3.6 & 3.7 \\ 67.5 & 67.7 \\ 46.7 & 46.4 \\ 53.3 & 53.6\end{array}$

necessary to derive answers (i.e. numeric recall or the judgement of which answer category best fits the desired answer; see Tourangeau et al. (2000)). We expect all three closed question formats to be answered faster than open questions, and fixed choice questions to be answered faster than those with multiple-response categories. We also expect items requiring numeric responses to take longer than fixed choice questions given the likely recall task that is involved. 
The field sequence number does not reflect the position of a particular question in the questionnaire but the actual number of questions answered by the respondent up to that particular field. Unlike the position of an item in the questionnaire this indicator matches the respondent's experience given the varying number of conditional questions and loops (e.g. a series of questions for each pregnancy or partner) that are asked of each respondent. The mean field sequence number in Table 1 clearly shows the difference in length (the number of items) between the female and male interviews.

Because reading and listening time are part of our time measure, the word count serves as an important control. A specific word count is difficult for questions that contain fill text-i.e. customized wording based on prior answers or respondent characteristics. Given that these vary across respondents, we could not derive the length of the fills for each item as administered to each respondent. Thus, the fill flag serves as a proxy measure for the additional text contained in fills.

Interviewer instructions and show cards both indicate actions that are needed to be taken by the interviewer. Although show cards add reading time (for the show card), interviewer instructions may be read in early administrations of the survey but used less as the interviewer becomes more familiar with the instrument.

Information on respondent characteristics was added from the NSFG cycle 6 questionnaire itself. Although the questionnaire contains plenty of data, we restricted ourselves to variables that were previously found to be related to response times. Respondents' age is correlated with cognitive decline and reduced working memory capacity (see Salthouse (1991) and Schwarz et al. (1999)). Respondents' education has been found to influence response times, with reduced times for more educated people (Salthouse, 1991; Yan and Tourangeau, 2008). Interviews that were conducted in Spanish have been found to take longer than those conducted in English (e.g. Wells et al. (2010)), so we use this rather than whether the respondent is of Hispanic origin. Marital status was added as a control, because of its close substantive relation to many of the NSFG questions.

Less is known about the effect of interviewer characteristics on response times. Similarly to the respondent characteristics, we expect age and education to play a role in the question administration speed. Similarly, we expect interviewer experience to be associated with response times, with more experienced interviewers being faster in administering the questionnaire (see Olson and Peytchev (2007)), reflecting familiarity with the task of administering survey questions on a computer. We had measures of both general interviewing experience and specific experience with CAI. Given that these variables are strongly correlated, preliminary analysis (which is not shown) led us to pick the latter for subsequent analyses.

We explore several interactions, e.g. to see whether respondent and interviewer education or age might interact in affecting item level times. Similarly we examine interactions between interviewer experience and field types, with the expectation that more experienced interviewers may have less difficulty with longer or more complex questions (e.g. those involving fills or interviewer instructions).

Our dependent variable measures the time that is spent on each field in the questionnaire. Time measures are recorded in milliseconds in the Blaise audit trails. To increase readability we report time in seconds. We report unadjusted measures of time without controlling for baseline speed, as is done in many response time studies. For one, the NSFG does not begin with a common set of questions asked of all respondents. Furthermore, in our statistical models we compare average response times for different items and are interested in identifying items with unusual performance rather than identifying respondents with unusual response times. Typical respondent features that affect baseline response time are captured through the demographic 
characteristics that were discussed above. The interviewer contribution to any variation in baseline speed is captured through a separate variance component, which we describe in Section 3.

\subsection{Data preparation}

A variety of data cleaning and exclusion activities were undertaken before the analysis. First, fields with unusually low and unusually high response times were removed from the analysis. Specifically, we removed fields with a response time that was shorter than $1 \mathrm{~s}$, given that it is not possible to administer a question in that short a time. As is common in response time analyses, fields with response times that are greater than 2 standard deviations above the mean (more than $172 \mathrm{~s}$ ), based on combined analyses for females and males, were also removed.

Second, missing data were present at all three levels. Most of the missing data are due to the lack of interviewer information. Over 260 interviewers were asked to complete and return a questionnaire after training, but not all did so. Among 256 interviewers who conducted at least one female interview, 54 did not complete the interviewer questionnaire (representing about $19.7 \%$ of the total fields). Five interviewers completed only one interview and were also excluded from the analyses to avoid confounding with respondent characteristics. Among 248 interviewers completing at least one male questionnaire, 47 did not complete the interviewer questionnaire (representing 19.1\% of the fields), and a further 11 completed only one interview. The field level and respondent level models did not differ on the basis of these exclusions, so we removed the cases without interviewer information from all models described below, to facilitate comparison of effects across models. On the respondent level we also lost some cases because of item missing data on some of the respondent covariates. A small number of completed interview cases did not have complete Blaise files. These were also excluded from our analyses, as were partial interviews (breakoffs).

Finally, we excluded for conceptual reasons fields that contained no questions (i.e. items for interviewer review or verification) and the household roster items (section A of the survey).

With all of these exclusions, the number of fields that were used in the analysis drops from 2.97 million for females and 1.25 million for males to 2.2 million and 870000 respectively. Table 2 shows the final count of observations that were used in subsequent analyses.

As is implied by Table 2, there is a hierarchy of observations, with items nested within respondents, and respondents nested within interviewers. A single interview contains an average of 354

Table 2. Number of observations available for response time analysis

\begin{tabular}{|lcc|}
\hline & $\begin{array}{c}\text { Results for } \\
\text { females }\end{array}$ & $\begin{array}{c}\text { Results } \\
\text { for males }\end{array}$ \\
\hline Number of fields (items) & 2155499 & 872500 \\
Number of respondents & 6096 & 3953 \\
Number of interviewers & 197 & 192 \\
Fields (items) per respondent & & \\
$\quad$ Mean & 353.59 & 220.72 \\
$\quad$ Minimum & 7 & 29 \\
Maximum & 755 & 391 \\
Respondents per interviewer & & \\
$\quad$ Mean & 30.94 & 20.59 \\
Minimum & 1 & 1 \\
Maximum & 147 & 103 \\
\hline
\end{tabular}


fields for the females file and 221 for the males file. The variation in the number of items per respondent is due to different filtering through the questionnaire.

\section{Analysis methods}

Unweighted multilevel linear mixed models (see West et al. (2007)) are used to explore the effect of the field indicators on response times. Such multilevel models allow for correlation in response times for items answered by the same respondent and respondents interviewed by the same interviewer. Failure to account for such clustering would probably underestimate the standard errors and bias inference that is made from the analyses. Furthermore, the multilevel models allow us to examine and control for the contribution of respondent and interviewer characteristics on response times. Our goal is to uncover and explore systematic variation in completion time that can be accounted for by characteristics of these three sources.

We first fit two (one for females and one for males) random-intercept-only models (null models), not including any covariates, to ascertain the proportion of variance that is accounted for by each of the levels in the hierarchy. The model can be specified as

$$
y_{i j k}=\beta_{0}+\mu_{k}+\mu_{j k}+\varepsilon_{i j k},
$$

where $y_{i j k}$ is the field time (expressed in seconds) for item $i$ nested within respondent $j$ interviewed by interviewer $k, \mu_{k}$ is the random effect that is associated with the interviewer, $\mu_{j k}$ is the random effect that is associated with the respondent and $\varepsilon_{i j k}$ is the residual variability that is associated with each item $i$, again nested within respondent $j$ interviewed by interviewer $k$. Again, all random effects are assumed to follow a normal distribution with $u_{j k} \sim N\left(0, \sigma_{u}^{2}\right)$ and $\varepsilon_{i j k} \sim N\left(0, \sigma_{\varepsilon}^{2}\right)$.

Using this, we estimate the intraclass correlation coefficient for each level of the model, as follows:

$$
\mathrm{ICC}_{k}=\frac{\sigma_{k}^{2}}{\sigma_{k}^{2}+\sigma_{j k}^{2}+\sigma_{i j k}^{2}}
$$

for interviewers and

$$
\mathrm{ICC}_{j k}=\frac{\sigma_{k}^{2}+\sigma_{j k}^{2}}{\sigma_{k}^{2}+\sigma_{j k}^{2}+\sigma_{i j k}^{2}}
$$

for respondents, where $\sigma_{k}^{2}$ is the variance of the random effects that are associated with interviewers, $\sigma_{j k}^{2}$ is the variance that is associated with respondents and $\sigma_{i j k}^{2}$ the variance that is associated with items. $\mathrm{ICC}_{k}$ is an estimate of the unexplained (random) variation in response time at the interviewer level, and $\mathrm{ICC}_{j k}$ is an estimate of the proportion of unexplained variation at the respondent level.

To explore the sources of this variation in more detail, we then fit three successive models for each of the females and males data sets, first examining field characteristics then adding respondent characteristics and finally adding interviewer characteristics. The full model can be expressed as

$$
y_{i j k}=\beta_{0}+\beta_{1} x_{i j k}+\beta_{2} v_{j k}+\beta_{3} z_{k}+\mu_{k}+\mu_{j k}+\varepsilon_{i j k},
$$

where $x_{i j k}$ are a vector of covariates at the field level, $v_{j k}$ are respondent level covariates and $z_{k}$ are interviewer level covariates. 
It should be noted that NSFG interviewers are largely assigned to a single primary sampling unit. Therefore variation at the interviewer level could also be interpreted as variation at the area level. Given that we control for respondent characteristics it is difficult to imagine which area level features - beyond the distinct composition of respondents within an area-would influence the response time. We thus interpret variation at the interviewer level as interviewer effects although some of this may be area level effects. We return to this point later.

All models were fitted by using the PROC MIXED procedure in SAS. Using restricted maximum likelihood estimation and maximum likelihood estimation yielded equivalent results (see West et al. (2007)). We present results of estimated coefficients by using the latter method. We use the Akaike information criterion (AIC) and the log-likelihood ratio tests for model comparison (McLachlan and Peel, 2000).

\section{Results}

\subsection{Model fit and estimated random effects}

The results of various specifications of random-intercept models without and with covariates (estimated random-effect components, intraclass correlations and model fit statistics) are presented in Table 3. From the intraclass correlations, it can be seen that interviewers contribute about $1.5 \%$ of the variation and respondents about $3.8 \%$ of the variation in the females model, with the corresponding values for the males model being $1.9 \%$ and $6.3 \%$ respectively. The intraclass correlations in Table 3 suggest that most of the variation $(96 \%$ for females and $94 \%$ for males) is at the individual field or item level, with respondents and interviewers accounting for modest amounts of the variation in field times.

Also presented in Table 3 are the AIC values as a measure of goodness of fit for each successive model. Reductions in the AIC show improvements in model fit. Examining the Bayes information criterion or log-likelihoods of the models (which are not shown) yields similar conclusions. Adding a set of fixed effects for field characteristics (see model 1) accounts for about $6 \%$ of the variation at the field level for females and about $11 \%$ for males (comparing $\sigma_{i j k}^{2}$ for model 1 with $\sigma_{i j k}^{2}$ for model 0 ). Likelihood ratio tests reveal that the addition of these covariates produces statistically significant $(p<0.0001)$ improvements in model fit.

Model 2 adds a set of respondent level fixed effects. As can be seen from Table 3, these variables again account for a modest proportion of the variation at the respondent level (about 13\% for females and about $14 \%$ for males). However, given the large number of cases, the model fit is significantly $(p<0.0001)$ improved over model 1 for both females and males.

Finally we add a set of interviewer level fixed effects to the models. Here the reductions in the residual interviewer level variance are modest, implying that not much of the variation due to interviewers is explained by the interviewer level variables. The likelihood ratio tests for the addition of these fixed effects are significant for females $(p=0.0065)$ but not for males $(p=0.082)$.

An examination of the coefficients for the fixed effects across the nested models reveals little change in values with the addition of the respondent and interviewer level effects. We thus present estimated coefficients for only the two final models, including covariates at all three levels (Table 4). We describe the effects of each set of variables below.

\subsection{Item or field characteristics}

Given the large number of observations, it is not surprising that all field level variables reach statistical significance in both the females and males models. Looking at the item level predictors, we can see from Table 4 that the length of the question (measured in the number of 
Table 3. Estimated variance components, intraclass correlations and model fit statistics for different specifications of the multilevel models for response time analysis for females and males (dependent variable: time on field in seconds)

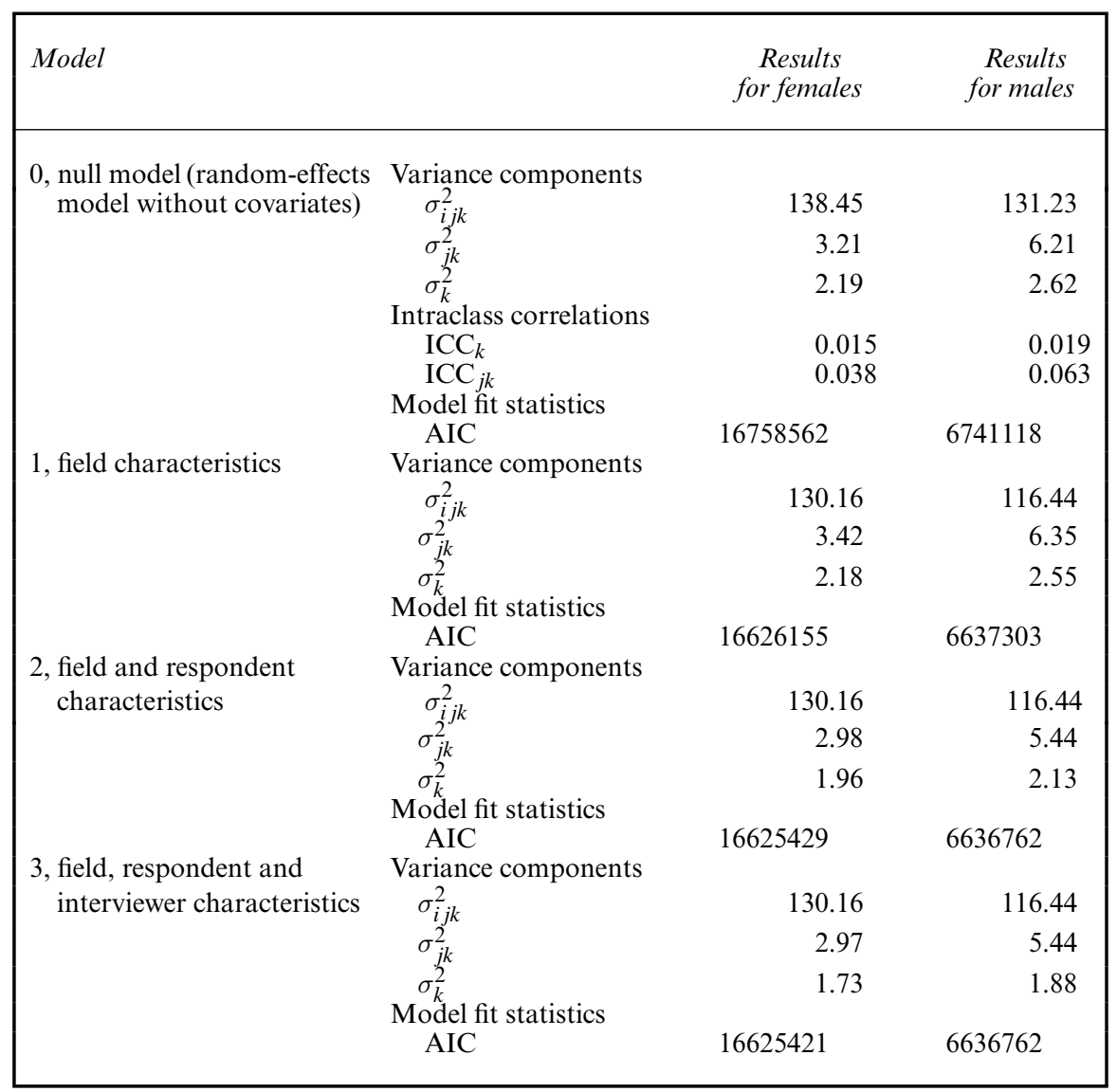

words) is positively associated with completion time, but this effect is relatively small, with each additional word adding about a 10th of a second to the time for females and just under a fifth of a second for males.

Turning next to field type, it is not surprising that items soliciting open responses (the reference category) take longer on average than other types of items. Items with multiple responses take less time than single-choice responses, controlling for the number of words in the field and other factors. This effect is more pronounced for females than for males, although the number of these types of field is relatively small. Fields requiring numeric (integer) entry (including dates) take longer than single- or multiple-response fields, suggesting that the recall and judgement process increases the response time.

The field sequence number is also positively associated with completion time, suggesting that the time to administer a question increases slightly over the course of the interview. This finding is somewhat unexpected, given the prevailing belief that interviewers speed up their delivery and respondents answer more quickly as the interview progresses. This may be a function of the fact that more difficult or demanding (i.e. cognitively complex or sensitive) questions are asked near the end of the survey. Beyond the simple word count, we do not have a covariate in the 
Table 4. Estimated final multilevel models for females and males including field, respondent and interviewer characteristics (dependent variable: time on field in seconds)

\begin{tabular}{|c|c|c|c|c|}
\hline \multirow[t]{2}{*}{ Effect } & \multicolumn{2}{|c|}{ Results for females } & \multicolumn{2}{|c|}{ Results for males } \\
\hline & Estimate & $\begin{array}{l}\text { Standard } \\
\quad \text { error }\end{array}$ & Estimate & $\begin{array}{l}\text { Standard } \\
\text { error }\end{array}$ \\
\hline Intercept & $8.08 \dagger$ & 0.55 & $5.81 \dagger$ & 0.65 \\
\hline \multicolumn{5}{|l|}{ Field or item characteristics } \\
\hline Word count & $0.089 \dagger$ & 0.00033 & $0.17 \dagger$ & 0.00065 \\
\hline \multicolumn{5}{|l|}{ Field type } \\
\hline Fixed choice & $-2.67 \dagger$ & 0.034 & $-0.32 \dagger$ & 0.042 \\
\hline Integer & $-1.98 \dagger$ & 0.037 & $-0.38 \dagger$ & 0.050 \\
\hline Multiple response & $-6.41 \dagger$ & 0.047 & $-0.88 \dagger$ & 0.080 \\
\hline Open & - & - & - & - \\
\hline Field sequence number & $0.0030 \dagger$ & 0.000060 & $0.0046 \dagger$ & 0.00026 \\
\hline ACASI & $-0.78 \dagger$ & 0.028 & $-2.57 \dagger$ & 0.045 \\
\hline Flag: instruction & $-1.02 \dagger$ & 0.025 & $-1.30 \dagger$ & 0.035 \\
\hline Flag: fill & $0.42 \dagger$ & 0.017 & $-0.32 \dagger$ & 0.026 \\
\hline Flag: show card & $6.12 \dagger$ & 0.026 & $4.46 \dagger$ & 0.042 \\
\hline Flag: help & $0.51 \dagger$ & 0.021 & $0.78 \dagger$ & 0.034 \\
\hline \multicolumn{5}{|l|}{ Respondent characteristics } \\
\hline \multicolumn{5}{|l|}{ Race } \\
\hline Black & $0.12 \dagger$ & 0.070 & $0.47 \dagger$ & 0.11 \\
\hline Other & $0.49 \$$ & 0.087 & $0.81 \dagger$ & 0.13 \\
\hline \multirow{2}{*}{\multicolumn{5}{|c|}{ Marital status }} \\
\hline & & & & \\
\hline Married & $-0.17 \hbar$ & 0.066 & $-0.37 \ddagger$ & 0.11 \\
\hline Cohabiting & -0.25 & 0.088 & -0.48 & 0.15 \\
\hline Formerly married & $-0.31 \dagger$ & 0.089 & -0.41 & 0.16 \\
\hline Never married & - & - & - & - \\
\hline \multicolumn{5}{|l|}{ Education } \\
\hline Less than high school & $1.43 \dagger$ & 0.084 & $2.12 \dagger$ & 0.14 \\
\hline High school graduate & $0.71 \dagger$ & 0.072 & $1.03 \dagger$ & 0.13 \\
\hline Some college & $0.31 \dagger$ & 0.071 & $0.43 \dagger$ & 0.13 \\
\hline College graduate & - & - & - & - \\
\hline Age & $0.021 \dagger$ & 0.0036 & $0.061 \dagger$ & 0.0059 \\
\hline Language of interview (1, English) & $-1.81 \dagger$ & 0.12 & $-2.13 \dagger$ & 0.19 \\
\hline \multicolumn{5}{|l|}{ Interviewer characteristics } \\
\hline \multicolumn{5}{|l|}{ Education } \\
\hline Unknown & 0.27 & 0.58 & 0.81 & 0.64 \\
\hline High school graduate & -0.18 & 0.31 & -0.12 & 0.34 \\
\hline College graduate & -0.49 & 0.30 & -0.49 & 0.33 \\
\hline Graduate school & - & - & - & - \\
\hline Spanish speaker $(1$, yes $)$ & $0.55 \S$ & 0.25 & $0.77 t$ & 0.28 \\
\hline Age & $0.031 \dagger$ & 0.0087 & 0.016 & 0.010 \\
\hline \multicolumn{5}{|l|}{ Race } \\
\hline Unknown & -0.11 & 0.57 & -0.046 & 0.65 \\
\hline Black & 0.11 & 0.25 & -0.064 & 0.29 \\
\hline Other & -0.00051 & 0.57 & 0.52 & 0.65 \\
\hline White & - & - & - & - \\
\hline CAI experience $(1$, yes $)$ & $-0.61 \dagger$ & 0.21 & -0.36 & 0.24 \\
\hline
\end{tabular}

$\dagger p<0.001$.

$\$ p<0.01$.

$\S p<0.05$. 
set of automatically derived item features that reflects the difficulty or sensitivity of an item. As a proxy for item content, we tested models with indicators for each of the sections (thematic groupings of items), rather than a linear measure of field sequence. Those models (which are not shown) also suggest considerable variation in response times across sections but do not markedly improve the fit over the models in Table 4.

It is also interesting to note that the self-administered ACASI items take less time (on average) to complete than the interviewer-administered items. The pace of the recorded ACASI voice files is fixed and is likely to be slower than the interviewers' pace (given that encouraging interviewers to slow down delivery is often emphasized in training). This suggests that respondents are not making full use of the audio files and are answering before the entire question has been read (see Couper et al. (2009)).

The remaining variables in Table 4 are flags for characteristics of questions that affect the response time. First, items containing interviewer instructions take less time on average than those that do not. This is somewhat surprising, as we expected that the presence of instructions indicated a more complex question or the need for further probing by the interviewer. This may also suggest that the interviewers may not be reading the instructions as intended.

As noted earlier, we created a flag for questions containing fills (customized question text) as we could not calculate the exact number of words for each question tailored to an individual respondent. Questions with fills take longer to administer than those without fills for females, but the opposite effect is found for males. One post hoc explanation is that the fills that are used for the male instrument are less complex than those for the female instrument, but this deserves further investigation. It may be that the type of fill (e.g. a specific date, a name or other previously reported information) may affect delivery time in different ways.

As we expected, questions with show cards take significantly longer to deliver $(6 \mathrm{~s}$ on average for females and $4.5 \mathrm{~s}$ on average for males). These tend to be longer, more complex questions, but this effect is controlling for the length of the question. This suggests that show cards are being used as designed, with respondents taking the time to review the answers that are provided. Finally, fields with question-by-question help take about half a second longer to administer than those without such help. We do not have an indicator for whether the help screen was actually accessed, but evidence from other studies suggests that the use of such help by interviewers is quite rare (e.g. Couper et al. (1997a)). This is another area for further exploration.

\subsection{Respondent characteristics}

The next set of variables in the models in Table 4 is respondent characteristics. We have already noted that the item level coefficients show little change with the addition of respondent and interviewer level effects. Our interest here is whether there is systematic variation in administration time by respondent characteristics, controlling for characteristics of the items.

We see that respondent race is significantly associated with response time, with minorities taking more time to answer than white respondents. Marital status is also significant for both females and males, with those who are never married taking longer than those who are currently or formerly married (controlling for age). This is somewhat surprising, as the latter groups are likely to have more complex family circumstances (which in turn would lead to a larger number of complex recall questions).

The findings for education and age are consistent with those reported by Yan and Tourangeau (2008) and support their hypothesis related to cognitive capacity. Older people and those with less education take longer to answer comparable questions than those who are younger or better educated. Similar results were reported by Couper et al. (2009) for the ACASI items.

Finally, the significant association of language of interview with time is consistent with the 
findings that have been reported elsewhere (e.g. Wells et al. (2010)). Surveys that are administered in Spanish take longer than those administered in English, in part because it takes more words to express the same ideas in Spanish than in English. Our word count variable is based on the English version of the instrument. We also tested a variable for whether the respondent identified as Hispanic or not. This was significantly associated with time but is highly correlated with the language of the interview, which is a more powerful predictor of time.

\subsection{Interviewer characteristics}

The final set of covariates in Table 4 relates to interviewer characteristics. As noted earlier, interviewers account for a relatively small proportion of the overall variation in response times, and adding fixed effects at the interviewer level does not explain much of this variation. This suggests that interviewers are having relatively little influence on completion times. We find that interviewers' education is not significantly related to completion time. In part this may reflect the fact that there is less variation in interviewers' education than in respondents' education. Interviewers who speak Spanish take about half a second longer to administer each item (controlling for language of the interview). Interviewers' age is associated with longer response times for female respondents but not for males (remember that all NSFG interviewers are female). The interviewer's race has no significant association with time for either females or males. Interviewers with prior CAI experience take less time on average (about 0.6 s) to administer the female instrument. The effect of CAI experience is in the same direction for males but does not reach significance.

\subsection{Interactions}

We explored several cross-level interactions (the results are not shown) but found few noteworthy effects. For example, respondent and interviewer education did not significantly interact in affecting response times ( $p=0.42$ for females and $p=0.35$ for males). Similarly, the interactions of interviewer and respondent age on completion times were not significant ( $p=0.31$ for females and $p=0.53$ for males). Several interactions of interviewer experience with field characteristics on response times reached significance in one model but not the other, and the effects were not readily interpretable.

\section{Discussion}

We have presented a set of analyses with item level time as the dependent variable, to explore the item, respondent and interviewer level correlates of survey administration time. These analyses suggest systematic covariation of characteristics at all three levels with the time that it takes to administer a survey item in the NSFG male and female instruments.

Our exploratory study has three major findings: first, automatically derived indicators of item characteristics are found to vary systematically with response time. The indicators that we created from the Blaise audit trail files predominantly captured syntactical features of survey items, e.g. the presence of fills and show cards, the available answer categories and word counts. Despite this, much of the variation in item level times remains unaccounted for.

Second, the results that are presented here for observational data from a large-scale production survey replicate findings on respondent characteristics and response times that have been found in prior (mostly CATI) studies, which were explicitly designed to study response times or in which response time measures were taken in a much more controlled way (e.g. explicitly timed by the interviewer).

Third, NSFG interviewers also appear to contribute independently to the completion times, 
although their measured demographic characteristics and experience explain only a small fraction of the interviewer variance component. The NSFG design does not allow us statistically to separate interviewer and area effects; thus part of the unexplained higher level variance components could be due to area effects.

Unfortunately the reliance in this study on item level characteristics that could be automatically derived from the CAPI questionnaire script limits the number of covariates on the item level available for analysis. Information about the content and nature of the questions asked were therefore not included in the models. Features such as the complexity of a question, length of recall periods or the sensitivity of particular items are known to affect response times. Such indicators, however, require human judgement or content analysis of the question text or respondent answers. Our goal here was to use automatically generated data that can be readily available without manual coding. Some respondent characteristics can serve as a proxy for some of these item features. For example, in the NSFG case, information on time since pregnancy could serve as a proxy for recall challenges in reporting details about that pregnancy. For the present analysis, only a limited number of respondent characteristics was available. In their absence there is no straightforward way to take the different filtering rules into account that probably resulted in differences with respect to the sensitivity and complexity of the questions asked.

Future work will need to explore the issue of why the presence of interviewer instructions is associated with faster completion times. To do so, it will be necessary to augment the automatically derived indicators with substantive information about the nature of the questions that are typically paired with interviewer instructions. Similarly, the finding that items with fills take more time to administer for females but less time for males needs to be examined further. The association of respondent race and marital status with completion time also suggests that there may be unmeasured item level covariates that need to be explored. Specifically, with regard to marital status, it may be that different respondents are being asked different questions based on marital status, and the differences between these items are not captured in our item level variables.

Despite the limitations in the set of automatically generated item features and some unanswered questions regarding the effect of the substantive nature of the survey questions, the replication of known effects with this large field-based data set provides encouragement for those who are interested in a more process-control-oriented approach to survey research. In addition to using these data to explore which features of the instrument, respondents and interviewers are associated with response time, residuals from these models could be used to identify items, respondents or interviewers who take much more (or less) time than expected (e.g. 2 standard deviations below the mean) and to investigate these cases further. The utility of these kinds of analyses for survey design and management lies in using the paradata to identify cases (items, respondents and interviewers) that are outliers efficiently ('out of control' in the statistical process control perspective), and targeting more expensive evaluation methods (such as coding and analysing digital recordings, conducting reinterviews and conducting laboratory-based or field experiments) at those cases. Items that behave in unexpected ways (i.e. take significantly less time or more time than expected, given their nature) could be subject to behaviour coding or cognitive laboratory testing. Respondents or interviews that are outliers could be subject to further data quality evaluations or verification that the prescribed protocol was followed. Similarly, interviewers who perform above or below control limits could have their work more carefully examined, potentially identifying falsified interviews or the need for retraining.

Likewise, if such models are fitted throughout the data collection period, and incoming data are compared with predicted values and expected distributions, interviewers could receive an alert when administering an item above or below certain time thresholds. For example, such 
alerts could help to prevent question deliveries that are too fast (given the age of the respective respondent), thereby indirectly reducing measurement error. Experimental research on using such interventions in the context of self-administered questionnaires has shown promising results (Conrad et al., 2005); similar experimental research is needed for interviewer-administered surveys.

The overall goal is to find ways to use existing data and paradata in an efficient manner to help to evaluate and improve the quality of survey data collection. This work represents one step in that direction.

\section{Acknowledgements}

The 2002 NSFG was carried out under a contract with the Centers for Disease Control and Prevention's National Center for Health Statistics (contract 200-2000-07001, Robert Groves, Project Director). We thank John van Hoewyk and Pat Berglund for assistance with the analyses. We also thank William D. Mosher for his review and comments on an earlier draft of the paper, and Gabriele Durrant, Stephanie Eckman and the reviewers for their helpful comments.

\section{References}

Ajzen, I., Nichols, A. J. and Driver, B. C. (1995) Identifying salient beliefs about leisure activities: frequency of elicitation versus response latency. J. Appl. Socl Psychol., 25, 1391-1410.

Bassili, J. N. (1996) The how and the why of response latency measurement in telephone surveys. In Answering Questions: Methodology for Determining Cognitive and Communicative Processes in Survey Research (eds N. Schwarz and S. Sudman), pp. 319-346. San Francisco: Jossey-Bass.

Bassili, J. N. (2003) The minority slowness effect: subtle inhibitions in the expression of views not shared by others. J. Personlty Socl Psychol., 84, 261-276.

Bassili, J.N. and Krosnick, J. A. (2000) Do strength-related attitude properties determine susceptibility to response effects?: new evidence from response latency, attitude extremity, and aggregate indices. Polit. Psychol., 21, 107132 .

Bassili, J. N. and Scott, B. S. (1996) Response latency as a signal to question problems in survey research. Publ. Opin. Q., 60, 390-399.

Cannell, C. F., Miller, P. V. and Oksenberg, L. (1981) Research on interviewing techniques. In Sociological Methodology (ed. S. Leinhardt), pp. 389-437. San Francisco: Jossey-Bass.

Caspar, R. A. and Couper, M. P. (1997) Using keystroke files to assess respondent difficulties with an ACASI instrument. Proc. Surv. Res. Meth. Sect. Am. Statist. Ass., 239-244.

Conrad, F., Broome, J., Benki, J., Groves, R., Kreuter, F. and Vannette, D. (2010) To agree or not to agree?: impact of interview speech and survey participation decisions. In Proc. Jt Statist. Meet. American Statistical Association, pp. 5979-5993. Alexandria: American Statistical Association.

Conrad, F., Couper, M., Tourangeau, R. and Galesic, M. (2005) Interactive feedback can improve the quality of responses in web surveys. Proc. Surv. Res. Meth. Sect. Am. Statist. Ass., 3835-3840.

Couper, M. P. (1998) Measuring survey quality in a CASIC environment. Jt Statist. Meet. American Statistical Association, Dallas, Aug.

Couper, M. P., Hansen, S. E. and Sadosky, S. A. (1997a) Evaluating interviewer performance in a CAPI survey. In Survey Measurement and Process Quality (eds L. Lyberg, P. Biemer, M. Collins, E. de Leeuw, C. Dippo, N. Schwarz and D. Trewin), pp. 267-285. New York: Wiley.

Couper, M. P., Horm, J. and Schlegel, J. (1997b) Using trace files to evaluate the National Health Interview Survey CAPI instrument. Proc. Surv. Res. Meth. Sect. Am. Statist. Ass., 825-829.

Couper, M. P., Tourangeau, R. and Marvin, T. (2009) Taking the audio out of audio-CASI. Publ. Opin. Q., 73, 281-303.

Draisma, S. and Dijkstra, W. (2004) Response latency and (para) linguistic expressions as indicators of response error. In Methods for Testing and Evaluating Survey Questionnaires (eds S. Presser, J. Rogthgeb, M. Couper, J. Lessler, E. Martin, J. Martin and E. Singer), pp. 131-147. Hoboken: Wiley.

Faust, M. E., Balota, D. A., Spieler, D. H. and Ferraro, F. R. (1999) Individual differences in informationprocessing rate and amount: implications for group differences in response latency. Psychol. Bull., 126, 777799.

Fazio, R. H. (1986) How do attitudes guide behavior? In The Handbook of Motivation and Cognition: Foundation of Social Behavior (eds R. M. Sorrentino and E. T. Higgins), pp. 204-243. New York: Guilford. 
Fazio, R. H. (1990) A practical guide to the use of response latency in social psychological research. In Review of Personality and Social Psychology, vol. 11, Research Methods in Personality and Social Research (eds C. Hendrick and M. S. Clark), pp. 74-97. Newbury Park: Sage.

Fazio, R. H. and Williams, C. J. (1986) Attitude accessibility as a moderator of the attitude-perception and attitude behavior relations: an investigation of the 1984 Presidential Election. J. Personlty Socl Psychol., 51, $505-514$.

Fisher, D. L. and Glaser, R. A. (1996) Molar and latent models of cognitive slowing: implications for aging, dementia, depression, development, and intelligence. Psychonom. Bull. Rev., 3, 458-480.

Groves, R. M. and Heeringa, S. G. (2006) Responsive design for household surveys: tools for actively controlling survey errors and costs. J. R. Statist. Soc. A, 169, 439-457.

Hansen, S. E. and Marvin, T. (2001) Reporting on item times and keystroke from Blaise audit trails. Int. Blaise User Group Meet., Washington DC. (Available from http://www.blaiseusers.org/2001/papers / Hansen_Marvin--IBUC_Paper_FINAL . pdf.)

Heerwegh, D. (2003) Explaining response latencies and changing answers using client-side paradata from a web survey. Socl Sci. Comput. Rev., 21, 360-373.

Huckfeldt, R., Levine, J., Morgan, W. and Sprague, J. (1998) Election campaigns, social communication, and the accessibility of perceived discussant preference. Polit. Behav., 20, 263-294.

Knowles, E. S. and Condon, C. A. (1999) Why people say 'yes': a dual-process theory of acquiescence. J. Personlty Socl Psychol., 77, 379-386.

Kreuter, F., Couper, M. P. and Lyberg, L. E. (2010) The use of paradata to monitor and manage survey data collection. In Proc. Jt Statist. Meet. American Statistical Association, pp. 282-296. Alexandria: American Statistical Association.

Kreuter, F. and Kohler, U. (2009) Analyzing contact sequences in call record data: potential and limitation of sequence indicators for nonresponse adjustment in the European Social Survey. J. Off. Statist., 25, $203-226$.

LaFlamme, F. (2009) Data collection research using paradata at Statistics Canada. Modernisation of Statistics Production Conf., Stockholm, Nov.

Lepkowski, J. M., Mosher, W. D., Davis, K. E., Groves, R. M., Van Hoewyk, J. and Willem, J. (2006) National Survey of Family Growth, Cycle 6: sample design, weighting, imputation, and variance estimation. Vitl Hlth Statist., 2, no. 142.

Mayerl, J., Sellke, P. and Urban, D. (2005) Analyzing cognitive processes in CATI surveys with response latencies: an empirical evaluation of the consequences of using different baseline speed measures. In Schriftenreihe des Instituts fuer Sozialwissenschaften der Universität Stuttgart, no. 2/20005.

Mayerl, J. and Urban, D. (2008) Antwortreaktionszeiten in Survey-Analysen. Wiesbaden: Verlag für Sozialwissenschaften.

McLachlan, G. and Peel, D. (2000) Finite Mixture Models. New York: Wiley.

Olson, K. and Peytchev, A. (2007) Effect of interviewer experience on interview pace and interviewer attitudes. Publ. Opin. Q., 71, 273-286.

Salthouse, T. A. (1991) Theoretical Perspectives on Cognitive Aging. Hillsdale: Erlbaum.

Schaeffer, N. C. and Maynard, D. M. (2006) Sounds of silence: response latency in cognitive assessments. A. Meet. American Association for Public Opinion Research, Montreal, May 18th-21st.

Schwarz, N., Park, D., Knäuper, B. and Sudman, S. (1999) Cognition, Aging, and Self-reports. Washington DC: Psychology Press.

Smith, E. E. (1968) Choice reaction time: an analysis of major theoretical positions. Psychol. Bull., 69, 77-110.

Smith, E. R., Fazio, R. H. and Ceijka, M. A. (1996) Accessible attitudes influence categorization of multiply categorizable objects. J. Personlty Socl Psychol., 71, 888-898.

Smith, E. R., and Lerner, M. (1986) Development of automatism of social judgments. J. Personlty Socl Psychol., 50, 246-259.

Statistics Netherlands (2002) Blaise Developer's Guide. Heerlen: Statistics Netherlands.

Stern, M. J. (2008) The use of client-side paradata in analyzing the effects of visual layout on changing responses in web surveys. Fld Meth., 20, 377-398.

Tourangeau, R. (1992) Context effects on responses to attitude questions: attitudes as memory structures. In Context Effects in Social and Psychological Research (eds N. Schwarz and S. Sudman), pp. 35-47. New York: Springer.

Tourangeau, R., Rips, L. and Rasinski, K. (2000) The Psychology of Survey Response. Cambridge: Cambridge University Press.

Wagner-Menghin, M. M. (2002) Towards the identification of non-scalable personality questionnaire respondents: taking response time into account. Psychol. Beitr., 44, 62-77.

Wells, T., Vidalon, M. and DiSogra, C. (2010) Differences in length of survey administration between Spanishlanguage and English-language survey respondents. Proc. Surv. Res. Meth. Sec. Am. Statist. Ass., 6186-6191.

West, B. T., Welch, K. B. and Galecki, A. T. (2007) Linear Mixed Models; a Practical Guide using Statistical Software. Boca Raton: Chapman and Hall.

Yan, T. and Tourangeau, R. (2008) Fast times and easy questions: the effects of age, experience and question complexity on web survey response times. Appl. Cogn. Psychol., 22, 51-68. 